\title{
Is it possible to predict the development of an entecavir resistance mutation in patients with chronic hepatitis $B$ in clinical practice?
}

\author{
Joon Yeul Nam and Jeong-Hoon Lee \\ Department of Internal Medicine and Liver Research Institute, Seoul National University College of Medicine, Seoul, Korea
}

Keywords: Chronic hepatitis B; Entecavir; Resistance; Lamivudine

\section{See Article on Page 323}

Potent nucleos(t)ide analogs (NAs) with a high genetic barrier, such as entecavir (ETV), tenofovir disoproxil fumarate (TDF), and tenofovir alafenamide fumarate, are strongly recommended as a front-line treatment for chronic hepatitis B (CHB) by current international practice guidelines. ${ }^{1-3}$ Among the potent NAs, there has been no detectable resistance to TDF after 6 years of therapy in CHB patients. ${ }^{4}$ However, clinical resistance to ETV has been reported in NA-naïve patients, although the incidence was as low as $1.2 \%$ at year $3 .{ }^{5}$ Since this study reported a low resistance rate for ETV, excluding the non-response groups at 48 and 96 weeks, the ETV-resistance rate might have been underestimated, as compared with that seen in real-world clinical practice.

Hepatitis B virus (HBV) exists in the form of quasispecies in patients with CHB. ${ }^{6}$ During ETV treatment in patients who experienced low-potency NAs, lamivudine (LAM)-resistant strains that might not be detected in a baseline mutation test if the proportion of LAM-resistant strains were relatively small could become the predominant strains because of positive selection by ETV, as they are less susceptible to ETV. ${ }^{7}$ According to the two-hit mechanism of ETV resistance, the positive selection of LAM-resistant strains by ETV acts like the first hit, and the second hit of an additional variant in these selected strains could easily lead to the development of ETV resistance. ${ }^{8}$ Of course, there may be another pathway for the development of entecavir resistance besides this stepwise manner. A simultaneous genetic mutation against ETV can also occur without a predeveloped LAM resistance, although a requirement to simultaneously develop multiple resistances contributes to the high genetic barrier to ETV. ${ }^{9}$ However, the clinical characteristics of patients with potential ETV resistance have not yet been completely elucidated in real-world practice.

In this issue of Clinical and Molecular Hepatology, a Korean real-world study by Kim et al. indicates that a higher viral load 6 months after antiviral therapy and failure to achieve a complete virologic response (CVR) during treatment with ETV were independently associated with ETV resistance..$^{10}$ In this study, patients were excluded if they were confirmed to have any genotypic resistance to NAs at baseline. Eight (3.1\%) out of 258 patients

\section{Abbreviations:}

$C H B$, chronic hepatitis $B ; C V R$, complete virologic response; ETV, entecavir; HBV, Hepatitis B virus; LAM, lamivudine; NAs, nucleos(t)ide analogs; TDF, tenofovir disoproxil fumarate

\section{Corresponding author: Jeong-Hoon Lee}

Department of Internal Medicine, Seoul National University Hospital, 101 Daehak-ro, Jongno-gu, Seoul 03080, Korea Tel: +82-2-2072-2228, Fax: +82-2-743-6701

Email: pindra@empal.com or JHLeeMD@gmail.com http://orcid.org/0000-0002-0315-2080 
developed a genotypic resistance to ETV during the follow-up period. Multivariate analyses showed a higher $\log _{10}$ HBV DNA level at month 6 and failure to achieve a CVR (defined as undetectable serum HBV DNA by polymerase chain reaction $[<116$ copies $/ \mathrm{mL}]$ 6 months after the initiation of ETV $0.5 \mathrm{mg}$ once daily therapy) were two independent risk factors of ETV resistance. These findings indicate that a rapid (within 6 months after the initiation of ETV therapy) virologic response and CVR were critical predictors of successful antiviral treatment with ETV, including continuous antiviral efficacy and no subsequent virologic breakthrough or genotypic resistance. The results of this study will help to determine which patients should be treated more carefully during ETV treatment in daily clinical practice.

Interestingly, previous NA exposure did not affect ETV resistance in this study, a finding that conflicts with those of other previous studies. ${ }^{11,12}$ In a prior Korean study, even though NA-resistance mutations before ETV treatment were not detected, the NAexposure group had an increased the risk of genotypic resistance to ETV and incidence of virologic breakthrough, as compared with the NA-naïve group." Regarding this difference from previous studies, Kim et al. speculated that some patients who had been exposed to NAs at their local clinic before the ETV treatment might have been included in the NA-naïve group and agreed that the NA exposure might still have been a more important factor for the development of the ETV resistance than the higher $\log _{10} \mathrm{HBV}$ DNA level at 6 months or failure to achieve a CVR. In addition, ETV monotherapy was reported to reduce serum HBV DNA levels by $6-7 \log _{10}$ during the first year of treatment. ${ }^{13}$ Thus, the definition of CVR in this study might have been a composite surrogate marker influenced by the baseline HBV DNA level, as well as the efficacy of the ETV treatment. For example, patients with high baseline HBV DNA levels could fail to achieve a CVR even if the HBV were efficiently suppressed by ETV. Moreover, it might have been necessary to adjust the interaction between a CVR and HBV DNA levels at month 6 in the multivariable analysis.

Until now, risk factors for developing ETV resistance were not fully elucidated. Therefore, there has been no consensus on atrisk patients or the follow-up schedule for patients with CHB during ETV treatment. The study results of Kim et al. provide some answers to this question. The most important adverse event of antiviral therapy is the emergence of drug resistance. Therefore, it is necessary for physicians to provide more careful follow-up of patients who were previously exposed to NA or have high baseline HBV DNA levels with detectable HBV DNA after 6 months of ETV treatment.

\section{Conflicts of Interest}

The author have no conflicts to disclose.

\section{REFERENCES}

1. Sarin SK, Kumar M, Lau GK, Abbas Z, Chan HL, Chen CJ, et al. Asian-Pacific clinical practice guidelines on the management of hepatitis B: a 2015 update. Hepatol Int 2016;10:1-98.

2. Terrault NA, Bzowej NH, Chang KM, Hwang JP, Jonas MM, Murad MH. AASLD guidelines for treatment of chronic hepatitis $B$. Hepatology. 2016;63:261-283.

3. European Association for the Study of the Liver. EASL 2017 Clinical Practice Guidelines on the management of hepatitis B virus infection. J Hepatology. 2017;67:370-398.

4. Kitrinos KM, Corsa A, Liu Y, Flaherty J, Snow-Lampart A, Marcellin P, et al. No detectable resistance to tenofovir disoproxil fumarate after 6 years of therapy in patients with chronic hepatitis B. Hepatology 2014;59:434-442.

5. Tenney DJ, Rose RE, Baldick CJ, Pokornowski KA, Eggers BJ, Fang $J$, et al. Long-term monitoring shows hepatitis $B$ virus resistance to entecavir in nucleoside-naïve patients is rare through 5 years of therapy. Hepatology 2009;49:1503-1514.

6. Lim SG, Cheng Y, Guindon S, Seet BL, Lee LY, Hu P, et al. Viral quasispecies evolution during hepatitis Be antigen seroconversion. Gastroenterology 2007;133:951-958.

7. Ijaz S, Arnold C, Dervisevic S, Mechurova J, Tatman N, Tedder RS, et al. Dynamics of lamivudine-resistant hepatitis B virus during adefovir monotherapy versus lamivudine plus adefovir combination therapy. J Med Virol 2008;80:1160-1170.

8. Lok AS, Zoulim F, Locarnini S, Bartholomeusz A, Ghany MG, Pawlotsky JM, et al. Antiviral drug-resistant HBV: Standardization of nomenclature and assays and recommendations for management. Hepatology 2007;46:254-265.

9. Suzuki F, Akuta N, Suzuki Y, Yatsuji H, Sezaki H, Arase Y, et al. Selection of a virus strain resistant to entecavir in a nucleoside-naive patient with hepatitis B of genotype H. J Clin Virol 2007;39:149152.

10. Kim HJ, Cho Y, Jeon W, Kim B. Clinical characteristics of patients with chronic hepatitis B who developed genotypic resistance to entecavir: Real-life experience. Clin Mol Hepatol 2017;23:323-330.

11. Lee JH, Cho Y, Lee DH, Lee M, Yoo JJ, Choi WM, et al. Prior exposure to lamivudine increases entecavir resistance risk in chronic hepatitis B Patients without detectable lamivudine resistance. Antimicrob Agents Chemother 2014;58:1730-1737.

12. Reijnders JG, Deterding K, Petersen J, Zoulim F, Santantonio T, Buti $M$, et al. Antiviral effect of entecavir in chronic hepatitis $B$ : influence of prior exposure to nucleos ( $t$ ) ide analogues. J Hepatol 2010;52:493-500. 
Joon Yeul Nam, et al. Prediction of ETV resistance

13. Chang TT, Gish RG, de Man R, Gadano A, Sollano J, Chao YC, et al. A comparison of entecavir and lamivudine for $\mathrm{HBeAg}$-positive chronic hepatitis B. N Engl J Med 2006;354:1001-1010. 